University of Wollongong

Research Online

Faculty of Engineering and Information

Faculty of Engineering and Information

Sciences - Papers: Part A

Sciences

2000

Influence of in-plane displacement and double-aperture orientation on slope fringe formation in double-shearing-aperture speckle interferometry

K F. Wang

Yangzhou University

A Kiet Tieu

University of Wollongong, ktieu@uow.edu.au

Enbang Li

University of Wollongong, enbang@uow.edu.au

Follow this and additional works at: https://ro.uow.edu.au/eispapers

Part of the Engineering Commons, and the Science and Technology Studies Commons

Research Online is the open access institutional repository for the University of Wollongong. For further information contact the UOW Library: research-pubs@uow.edu.au 


\title{
Influence of in-plane displacement and double-aperture orientation on slope fringe formation in double-shearing-aperture speckle interferometry
}

\begin{abstract}
The influence of the in-plane displacement and double-aperture orientation on the slope fringe formation in double-shearing-aperture speckle interferometry is discussed in detail. The research results show that the two in-plane displacement components, one parallel to and the other perpendicular to the shearing direction, have an influence on the slope fringe formation and that the double-aperture orientation also has an important influence on the slope fringe formation. A theoretical analysis and experimental results are presented. The experimental results are in good agreement with the theoretical analysis.
\end{abstract}

\section{Keywords}

speckle, formation, fringe, interferometry, slope, influence, orientation, aperture, double, displacement, plane, shearing

Disciplines

Engineering | Science and Technology Studies

\section{Publication Details}

Wang, K. F., Tieu, A. K. \& Li, E. B. (2000). Influence of in-plane displacement and double-aperture orientation on slope fringe formation in double-shearing-aperture speckle interferometry. Optical Engineering, 39 (8), 2124-2128. 


\section{Influence of in-plane displacement and double-aperture orientation on slope fringe formation in double-shearing-aperture speckle interferometry}

\author{
K. F. Wang \\ Yangzhou University \\ Department of Mechanical Engineering \\ Engineering College \\ Yangzhou, Jiangsu 225009 \\ China
}

\author{
A. K. Tieu \\ E. B. Li \\ University of Wollongong \\ Department of Mechanical Engineering \\ Faculty of Engineering \\ Wollongong, NSW 2522 \\ Australia
}

\begin{abstract}
The influence of the in-plane displacement and doubleaperture orientation on the slope fringe formation in double-shearingaperture speckle interferometry is discussed in detail. The research results show that the two in-plane displacement components, one parallel to and the other perpendicular to the shearing direction, have an influence on the slope fringe formation and that the double-aperture orientation also has an important influence on the slope fringe formation. A theoretical analysis and experimental results are presented. The experimental results are in good agreement with the theoretical analysis. (c) 2000 Society of Photo-Optical Instrumentation Engineers. [S0091-3286(00)01908-5]
\end{abstract}

Subject terms: double-shearing-aperture speckle interferometry; slope fringe formation; in-plane displacement; double-aperture orientation.

Paper 990405 received Oct. 18, 1999, revised manuscript received Feb. 10, 2000; accepted for publication Feb. 10, 2000.

\section{Introduction}

The determination of slope distribution information is of vital importance in the investigation of the out-of-plane deformation of thin plates because the slope distribution is related to the stress and strain distributions of these plates. Speckle shearing interferometry is a powerful tool in the measurement of slope distributions and has been used extensively to analyze the stress and strain fields of thin plates. ${ }^{1,2}$

In speckle shearing interferometry, the slope distribution information concerning thin plates can be obtained using either single-aperture or double-aperture speckle shearing interferometry. With double-aperture speckle shearing interferometry, the contrast of fringes is good compared with that obtained in single-aperture speckle shearing interferometry, but double-aperture speckle shearing interferometry is sensitive to the in-plane displacement components ${ }^{3-6}$ and to the in-plane strain components. ${ }^{4-6}$ In Ref. 3, the influence of the in-plane displacement components on the slope fringe formation in speckle grating-shearing interferometry was first studied. The research result shows that the in-plane displacement components do have an influence on the slope fringe formation in double-aperture speckle grating-shearing interferometry. In Refs. 4 to 6, the influence of the in-plane displacement components on the slope fringe formation in multiaperture speckle wedge-shearing interferometry was also investigated. We can see from the results that the in-plane displacement components have an important influence on the slope fringe formation in multiaperture wedge-shearing interferometry.

In this paper, we discuss in detail the influence of the in-plane displacement and double-shearing-aperture orientation on the slope fringe formation in double-shearingaperture speckle interferometry, which, to our knowledge, has not been previously investigated. A quantitative analysis and experimental results are presented.

\section{Experimental Arrangement}

The experimental arrangement is shown in Fig. 1. A collimated $\mathrm{He}-\mathrm{Ne}$ laser beam normally illuminates the diffuse surface of an object. An imaging lens is focused on the diffuse surface. A mask with two apertures $A_{1}$ and $A_{2}$ drilled along the direction with an included angle $\theta$ with respect to the $x$ axis is symmetrically placed in front of the imaging lens. The scattered wavefront from the diffuse surface is divided into two wavefronts through the mask. Two shearing wedges with the same wedge angle and refractive index are axisymmetrically placed in front of apertures $A_{1}$ and $A_{2}$, respectively, with the shearing direction of each shearing wedge parallel to the $x$ axis. This experimental arrangement brings the two wavefronts coming from a point at the object plane to focus at two different points on the image plane. In other words, the wavefronts from two object points $O_{1}\left(x+\Delta_{o}, y\right)$ and $O_{1}\left(x-\Delta_{o}, y\right)$ through apertures $A_{1}$ and $A_{2}$, respectively, are added coherently at the image plane of the imaging system, as shown in Fig. 1. The equivalent shifting magnitude of each image at the object plane is given as follows:

$\Delta_{o}=d_{o}(\mu-1) \alpha$,

where $d_{o}$ is the object distance of the imaging system, $\mu$ is the refractive index of the shearing wedges, and $\alpha$ is the wedge angle. With this configuration, two exposure records, one before and the other after object deformation, are made on a single photographic plate. 


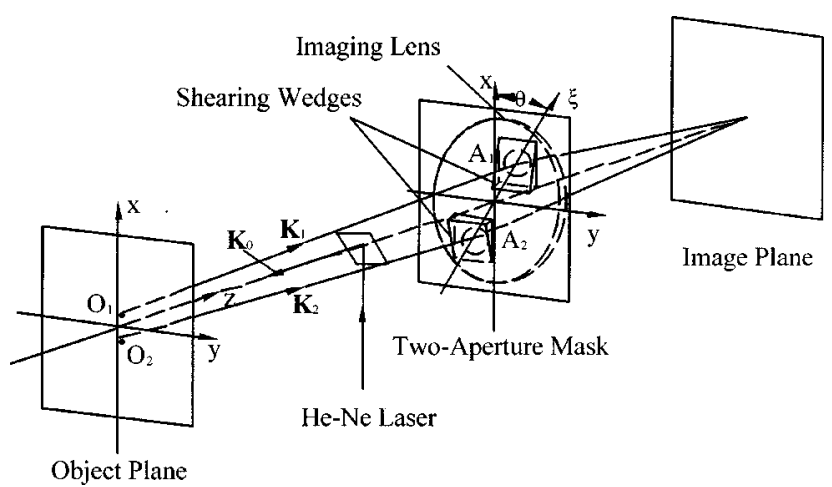

Fig. 1 Schematic of the recording system.

\section{Theoretical Analysis}

\subsection{Specklegram Record}

Assuming that the two scattered wavefronts from the object plane through apertures $A_{1}$ and $A_{2}$ have amplitudes $a_{1}$ and $a_{2}$, respectively, the total amplitude distribution at the image plane before deformation can be expressed as

$U_{1}=a_{1} \exp \left[i\left(\varphi_{1}+\beta\right)\right]+a_{2} \exp \left[i\left(\varphi_{2}-\beta\right)\right]$,

where $\varphi_{1}$ and $\varphi_{2}$ are the random phases corresponding respectively to the two object points $O_{1}\left(x+\Delta_{o}, y\right)$ and $O_{1}\left(x-\Delta_{o}, y\right)$, and $\beta$ is the phase corresponding to the grating-like structure in speckles introduced by the doubleaperture separation. The intensity distribution at the image plane before deformation is given by

$I_{1}=\left|U_{1}\right|^{2}=a_{1}^{2}+a_{2}^{2}+2 a_{1} a_{2} \cos \left(\varphi_{12}+2 \beta\right)$,

where $\varphi_{12}=\varphi_{1}-\varphi_{2}$ is the difference of random phases.

Similarly, the total amplitude distribution at the image plane after the object is deformed can be written as

$U_{2}=a_{1} \exp \left[i\left(\varphi_{1}+\delta_{1}+\beta\right)\right]+a_{2} \exp \left[i\left(\varphi_{2}+\delta_{2}-\beta\right)\right]$,

where $\delta_{1}$ and $\delta_{2}$ are the phase changes of the two wavefronts caused by object deformation. According to the directions of illumination and observation, the phase changes can be expressed as follows:

$$
\left\{\begin{array}{l}
\delta_{1}=\left(\mathbf{K}_{1}-\mathbf{K}_{0}\right) \cdot \mathbf{L}\left(x+\Delta_{o}, y\right)=\left(\mathbf{K}_{1}-\mathbf{K}_{0}\right) \cdot\left[\mathbf{L}(x, y)+\frac{\partial \mathbf{L}(x, y)}{\partial x} \Delta_{o}\right] \\
\delta_{2}=\left(\mathbf{K}_{2}-\mathbf{K}_{0}\right) \cdot \mathbf{L}\left(x-\Delta_{o}, y\right)=\left(\mathbf{K}_{2}-\mathbf{K}_{0}\right) \cdot\left[\mathbf{L}(x, y)-\frac{\partial \mathbf{L}(x, y)}{\partial x} \Delta_{o}\right]
\end{array}\right.
$$

where $\mathbf{K}_{0}$ is the propagation vector along the illumination direction, $\mathbf{K}_{1}$ and $\mathbf{K}_{2}$ are the propagation vectors along the observation directions, and $\mathbf{L}\left(x+\Delta_{o}, y\right)$ and $\mathbf{L}\left(x-\Delta_{o}, y\right)$ are the displacement vectors at the two object points $O_{1}\left(x+\Delta_{o}, y\right)$ and $O_{1}\left(x-\Delta_{o}, y\right)$, respectively, as shown in Fig. 1. The intensity distribution at the image plane of the imaging system after object deformation is given by
$I_{2}=\left|U_{2}\right|^{2}=a_{1}^{2}+a_{2}^{2}+2 a_{1} a_{2} \cos \left(\varphi_{12}+\delta_{12}+2 \beta\right)$,

where $\delta_{12}=\delta_{1}-\delta_{2}$ is the difference of the phase changes.

According to Eq. (5), $\delta_{12}$ can be expressed as

$$
\delta_{12}=\left(\mathbf{K}_{1}+\mathbf{K}_{2}-2 \mathbf{K}_{0}\right) \cdot \frac{\partial \mathbf{L}(x, y)}{\partial x} \Delta_{o}+\left(\mathbf{K}_{1}-\mathbf{K}_{2}\right) \cdot \mathbf{L}(x, y),
$$

where the displacement vector $\mathbf{L}(x, y)$ and its partial derivative vector $\partial \mathbf{L}(x, y) / \partial x$ can be given by

$$
\left\{\begin{array}{l}
\mathbf{L}(x, y)=u(x, y) \mathbf{i}+v(x, y) \mathbf{j}+w(x, y) \mathbf{k}, \\
\frac{\partial \mathbf{L}(x, y)}{\partial x}=\frac{\partial u(x, y)}{\partial x} \mathbf{i}+\frac{\partial v(x, y)}{\partial x} \mathbf{j}+\frac{\partial w(x, y)}{\partial x} \mathbf{k}
\end{array}\right.
$$

where $\mathbf{i}, \mathbf{j}$, and $\mathbf{k}$ are the unit vectors along the $x, y$, and $z$ directions, respectively. The propagation vectors $\mathbf{K}_{0}, \mathbf{K}_{1}$, and $\mathbf{K}_{2}$ in Eq. (7) can also be given, according to Fig. 1, by

$$
\left\{\begin{array}{l}
\mathbf{K}_{0}=\frac{2 \pi}{\lambda}\left[-\sin \gamma_{0}\left(\mathbf{i} \cos \theta_{0}+\mathbf{j} \sin \theta_{0}\right)-\mathbf{k} \cos \gamma_{0}\right], \\
\mathbf{K}_{1}=\frac{2 \pi}{\lambda}\left[\sin \gamma_{1}(\mathbf{i} \cos \theta+\mathbf{j} \sin \theta)+\mathbf{k} \cos \gamma_{1}\right], \\
\mathbf{K}_{2}=\frac{2 \pi}{\lambda}\left[-\sin \gamma_{2}(\mathbf{i} \cos \theta+\mathbf{j} \sin \theta)+\mathbf{k} \cos \gamma_{2}\right],
\end{array}\right.
$$

where $\theta$ is the included angle between the double-aperture direction and the $x$ axis. In the experimental arrangement in Fig. $1, \gamma_{1}, \gamma_{2} \ll 1$, which results in

$$
\left\{\begin{array}{l}
\sin \gamma_{1} \approx \sin \gamma_{2} \approx \frac{D}{2 d_{o}}, \\
\cos \gamma_{1} \approx \cos \gamma_{2} \approx 1,
\end{array}\right.
$$

where $D$ is the double-aperture separation. Therefore Eq. (9) can reduce to

$$
\left\{\begin{array}{l}
\mathbf{K}_{0}=\frac{2 \pi}{\lambda}\left[-\sin \gamma_{0}\left(\mathbf{i} \cos \theta_{0}+\mathbf{j} \sin \theta_{0}\right)-\mathbf{k} \cos \gamma_{0}\right], \\
\mathbf{K}_{1}=\frac{2 \pi}{\lambda}\left[\frac{D}{2 d_{o}}(\mathbf{i} \cos \theta+\mathbf{j} \sin \theta)+\mathbf{k}\right] \\
\mathbf{K}_{2}=\frac{2 \pi}{\lambda}\left[-\frac{D}{2 d_{o}}(\mathbf{i} \cos \theta+\mathbf{j} \sin \theta)+\mathbf{k}\right]
\end{array}\right.
$$

By substitution of Eqs. (8) and (11) into Eq. (7), the following equation can be obtained 


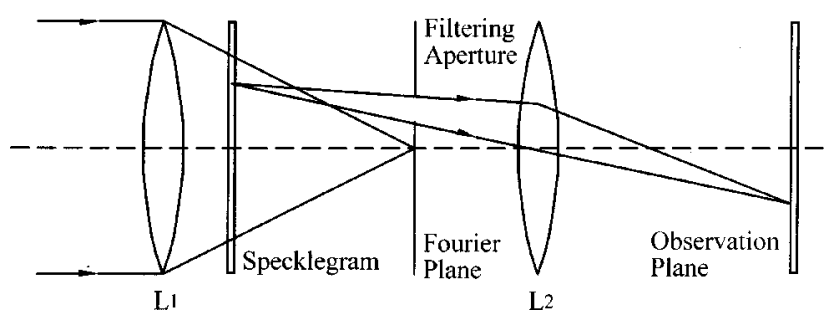

Fig. 2 Schematic of the analysis system.

$$
\begin{aligned}
\delta_{12} \approx & \frac{2 \pi}{\lambda} 2 \Delta_{o}\left[\sin \gamma_{0}\left(\mathbf{i} \cos \theta_{0}+\mathbf{j} \sin \theta_{0}\right)+\mathbf{k}\left(1+\cos \gamma_{0}\right)\right] \\
& \cdot \frac{\partial \mathbf{L}(x, y)}{\partial x}+\frac{2 \pi}{\lambda} \frac{D}{d_{o}}(\mathbf{i} \cos \theta+\mathbf{j} \sin \theta) \cdot \mathbf{L}(x, y) \\
= & \frac{2 \pi}{\lambda} 2 \Delta_{o}\left(1+\cos \gamma_{0}\right) \frac{\partial w(x, y)}{\partial x} \\
& +\frac{2 \pi}{\lambda} 2 \Delta_{o} \sin \gamma_{0}\left[\frac{\partial u(x, y)}{\partial x} \cos \theta_{0}+\frac{\partial v(x, y)}{\partial x} \sin \theta_{0}\right] \\
& +\frac{2 \pi}{\lambda} \frac{D}{d_{o}}[u(x, y) \cos \theta+v(x, y) \sin \theta]
\end{aligned}
$$

We can see from the preceding equation that the two inplane displacement components $u(x, y)$ and $v(x, y)$ have an influence on the slope fringe formation and that the doubleaperture orientation $\theta$ has also an important influence on the slope fringe formation. We can also see that the inplane strain components $\delta u(x, y) / \partial x$ and $\delta v(x, y) / \partial x$ and the illumination angles $\theta_{0}$ and $\gamma_{0}$ influence the slope fringe formation, but when utilizing normal illumination, $\gamma_{0}=0$, the in-plane strain components and the illumination direction have no influence on the slope fringe formation, and then Eq. (12) can reduce to

$$
\begin{aligned}
\delta_{12} \approx & \frac{2 \pi}{\lambda} 4 \Delta_{o} \frac{\partial w(x, y)}{\partial x}+\frac{2 \pi}{\lambda} \frac{D}{d_{o}}[u(x, y) \cos \theta \\
& +v(x, y) \sin \theta] .
\end{aligned}
$$

\subsection{Filtering Analysis}

The total intensity distribution recorded at the image plane of the imaging system as a result of double exposure records is expressed as

$$
\begin{aligned}
I_{t}= & I_{1}+I_{2} \\
= & 2\left(a_{1}^{2}+a_{2}^{2}\right)+2 a_{1} a_{2}\left[\cos \left(\varphi_{12}+2 \beta\right)\right. \\
& \left.+\cos \left(\varphi_{12}+\delta_{12}+2 \beta\right)\right] .
\end{aligned}
$$

To yield information fringes, the preceding double-exposed specklegram is placed in the Fourier filtering system as shown in Fig. 2. Three diffraction halos are formed at the Fourier plane of lens $L_{1}$ when subjected to Fourier filtering. The amplitude distribution at the observation plane of the analysis system by filtering via one of the two first-order halos is represented by
$U_{f}=a_{1} a_{2}\left\{\exp \left[i\left(\varphi_{12}+2 \beta\right)\right]+\exp \left[i\left(\varphi_{12}+\delta_{12}+2 \beta\right\}\right.\right.$,

and the intensity distribution at the observation plane is given by

$I_{f}=\left|U_{f}\right|^{2}=2 a_{1}^{2} a_{2}^{2}\left(1+\cos \delta_{12}\right)$.

We can see from Eqs. (13) and (16) that dark fringes will appear when $\delta_{12}=(2 n+1) \pi$, that is,

$$
\begin{gathered}
\frac{\partial w(x, y)}{\partial x}+\frac{D}{4 \Delta_{o} d_{o}}[u(x, y) \cos \theta+v(x, y) \sin \theta] \\
=\frac{(2 n+1) \lambda}{8 \Delta_{o}} \quad(n=0, \pm 1, \pm 2, \ldots),
\end{gathered}
$$

where $\lambda$ is the laser wavelength. Similarly bright fringes will appear when $\delta_{12}=2 n \pi$, that is,

$$
\begin{gathered}
\frac{\partial w(x, y)}{\partial x}+\frac{D}{4 \Delta_{o} d_{o}}[u(x, y) \cos \theta+v(x, y) \sin \theta] \\
=\frac{n \lambda}{4 \Delta_{o}} \quad(n=0, \pm 1, \pm 2, \ldots) .
\end{gathered}
$$

\section{Experimental Results}

The experiments were carried out using an experimental arrangement like that shown in Fig. 1. An aluminum plate with a radius of $26 \mathrm{~mm}$ and a thickness of $3 \mathrm{~mm}$ was used as the specimen at the object plane. The specimen was rigidly clamped along its edge and subjected to both an out-of-plane deformation by centrally loading at its center and an in-plane displacement by rotating around its center. The specimen surface was normally illuminated with a collimated beam from a 30-mW He-Ne laser (laser wavelength $\lambda=632.8 \mathrm{~nm}$ ) by placing a beamsplitter between the object plane and imaging lens, as shown in Fig. 1. The imaging lens had a focal distance of $240 \mathrm{~mm}$ and the imaging system had a magnification of 1.0. Two shearing wedges with the same wedge angle of $28 \mathrm{~min}$ and the same refractive index of 1.515 were axisymmetrically placed in front of a double-aperture mask with an aperture separation of 20 $\mathrm{mm}$.

According to Ref. 7, the deflection distribution of a circular plate, which is loaded at its center and rigidly clamped along its boundary, is given as follows:

$$
\begin{aligned}
w(x, y)= & w_{\max }\left(1-\frac{x^{2}+y^{2}}{r^{2}}+\frac{x^{2}+y^{2}}{r^{2}} \ln \frac{x^{2}+y^{2}}{r^{2}}\right) \\
& {\left[\left(x^{2}+y^{2}\right)^{1 / 2} \leqslant r\right], }
\end{aligned}
$$

where $w_{\max }$ and $r$ are the central deflection and radius of plate, respectively. According to the preceding equation, the equation of slope distribution is expressed as follows:

$\frac{\partial w(x, y)}{\partial x}=\frac{2 w_{\max }}{r} \frac{x}{r} \ln \frac{x^{2}+y^{2}}{r^{2}} \quad\left[\left(x^{2}+y^{2}\right)^{1 / 2} \leqslant r\right]$. 
In addition, the in-plane displacement components respectively along the $x$ and $y$ directions, when subjected to an in-plane rotation around its center, can be given by

$u(x, y)=\phi y, \quad v(x, y)=-\phi x \quad\left[\left(x^{2}+y^{2}\right)^{1 / 2} \leqslant r\right]$

where $\phi$ is the in-plane rotation angle of the specimen. By substitution of Eqs. (20) and (21) into Eqs. (13) and (18), we can obtain the following equations:

$$
\begin{aligned}
\delta_{12}= & \frac{2 \pi}{\lambda} 4 \Delta_{o} \frac{2 w_{\max }}{r}\left(\frac{x}{\dot{r}} \ln \frac{x^{2}+y^{2}}{r^{2}}\right. \\
& \left.+\frac{D \phi r^{2}}{8 \Delta_{o} d_{o} w_{\max }} \frac{y \cos \theta-x \sin \theta}{r}\right) \quad\left[\left(x^{2}+y^{2}\right)^{1 / 2} \leqslant r\right] .
\end{aligned}
$$

and

$$
\begin{aligned}
& \frac{x}{r} \ln \frac{x^{2}+y^{2}}{r^{2}}+\frac{D \phi r^{2}}{8 \Delta_{o} d_{o} w_{\max }} \frac{y \cos \theta-x \sin \theta}{r} \\
& \quad=\frac{n \lambda r}{8 \Delta_{o} w_{\max }} \quad\left[\left(x^{2}+y^{2}\right)^{1 / 2} \leqslant r, \quad n=0, \pm 1, \pm 2, \ldots\right] .
\end{aligned}
$$

Experiment I. The specimen was subjected to both an out-of-plane deflection of $5 \mu \mathrm{m}$ at its center and an in-plane rotation of $2 \mathrm{~min}$ around its center. The double-aperture orientation and shearing direction were parallel to the $x$ axis. By substitution of the experimental parameters into Eqs. (22) and (23), we obtain

$$
\begin{aligned}
\delta_{12}= & \frac{2 \pi}{\lambda} 4 \Delta_{o} \frac{2 w_{\max }}{r}\left(\frac{x}{r} \ln \frac{x^{2}+y^{2}}{r^{2}}+0.2036 \frac{y}{r}\right) \\
& {\left[\left(x^{2}+y^{2}\right)^{1 / 2} \leqslant r\right], }
\end{aligned}
$$

and

$$
\begin{gathered}
\frac{x}{r} \ln \frac{x^{2}+y^{2}}{r^{2}}+0.2036 \frac{y}{r}=0.2044 n \quad\left[\left(x^{2}+y^{2}\right)^{1 / 2} \leqslant r ;\right. \\
n=0, \pm 1, \pm 2, \ldots] .
\end{gathered}
$$

Experiment II. The specimen was subjected to an out-ofplane deflection of $5 \mu \mathrm{m}$ at its center and an in-plane rotation of $10 \mathrm{~min}$ around its center. The double-aperture orientation was perpendicular to the $x$ axis, while the shearing direction was still parallel to the $x$ axis. By substitution of the experimental parameters into Eqs. (22) and (23), we obtain

$$
\begin{aligned}
\delta_{12}= & \frac{2 \pi}{\lambda} 4 \Delta_{o} \frac{2 w_{\max }}{r}\left(\frac{x}{r} \ln \frac{x^{2}+y^{2}}{r^{2}}-1.018 \frac{x}{r}\right) \\
& {\left[\left(x^{2}+y^{2}\right)^{1 / 2} \leqslant r\right], }
\end{aligned}
$$

and

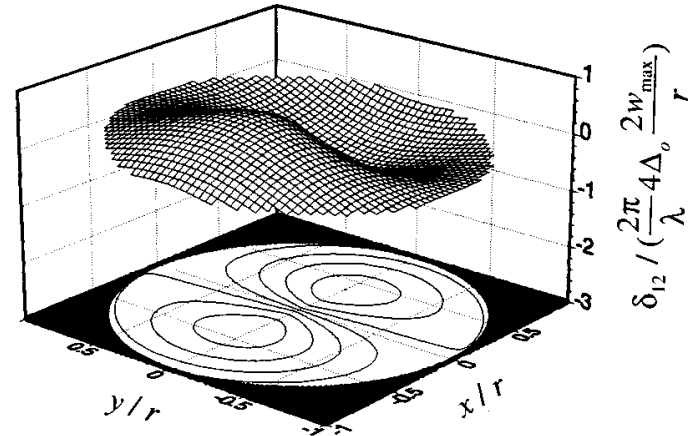

(a)

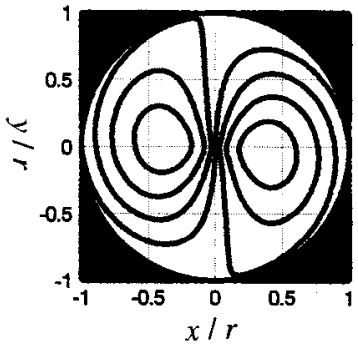

(b)

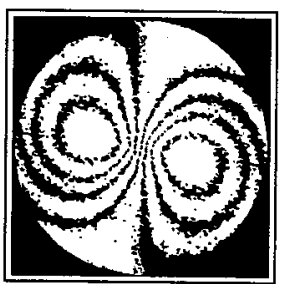

(c)
Fig. 3 Theoretical and experimental results for experiment I: (a) 3-D phase distribution and its contour projection, (b) 2-D distribution of contours, and (c) experimental result.

$$
\begin{gathered}
\frac{x}{r} \ln \frac{x^{2}+y^{2}}{r^{2}}-1.018 \frac{x}{r}=0.2044 n \quad\left[\left(x^{2}+y^{2}\right)^{1 / 2} \leqslant r ;\right. \\
n=0, \pm 1, \pm 2, \ldots] .
\end{gathered}
$$

The patterns of 3-D phase distributions corresponding to experiments I and II, which are drawn according to Eqs. (24) and (26), are shown in Figs. 3(a) and 4(a), respectively. The contours of phase distributions in Figs. 3(a) and 3(b), and in Figs. 4(a) and 4(b) are, respectively, drawn according to Eqs. (25) and (27).

The experimental results for experiments I and II are shown in Figs. 3(c) and 4(c), respectively. We can see that there is hardly any difference between Figs. 3(b) and 3(c), and between Figs. 4(b) and 4(c) with respect to fringe locations and shapes.

\section{Conclusions}

The influence of the in-plane displacement and doubleaperture orientation on the slope fringe formation in double-shearing-aperture speckle interferometry was discussed in detail. The research results show that all two in-plane displacement components, one parallel to and the other perpendicular to the shearing direction, have an influence on the slope fringe formation and that the doubleaperture orientation has also an important influence on the slope fringe formation. It is also shown that the in-plane strain components and the illumination direction have no influence on the slope fringe formation when the object surface is normally illuminated and the imaging system axisymmetrical. We can see from Figs. 3 and 4, respectively, that the experimental results are in good agreement with the quantitative analysis. 


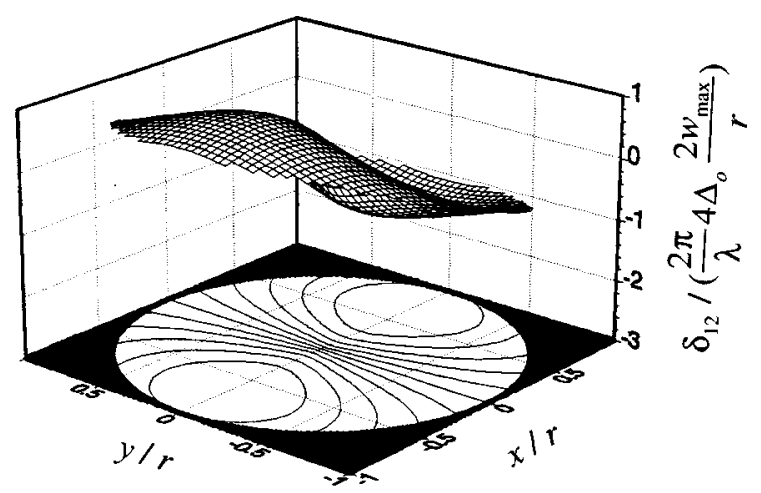

(a)
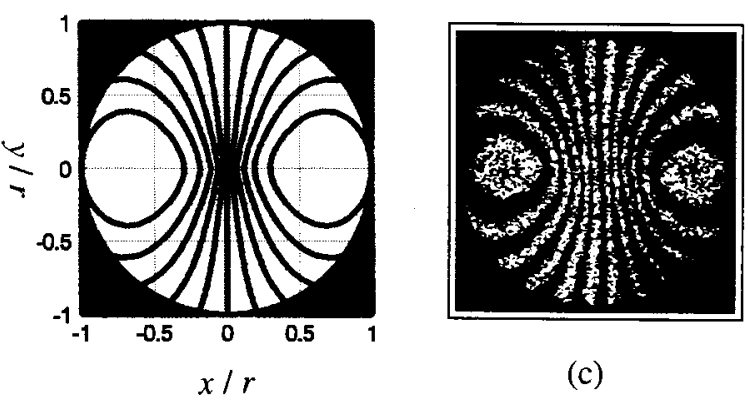

(b)

Fig. 4 Theoretical and experimental results for experiment II: (a) 3-D phase distribution and its contour projection, (b) 2-D distribution of contours, and (c) experimental result.

\section{Acknowledgments}

One of the authors (K. F. Wang) would like to thank the Education Commission of Jiangsu Province for providing financial support for his research work at University of Wollongong.

\section{References}

1. Y. Y. Hung and C. E. Taylor, "Measurement of slope of structura deflections by speckle-shearing interferometry," Exp. Mech. 14, 281 (1974).

2. Y. Y. Hung and C. Y. Liang, "Image-shearing camera for direct measurement of surface strain," Appl. Opt. 18, 1046 (1980).

3. Y. Iwahashi, K. Iwata, and R. Nagata, "Influence of in-plane displacement in single-aperture and double-aperture speckle shearing interferometry,"' Appl. Opt. 24, 2189 (1985).
4. N. K. Mohan, P. J. Masalkar, V. M. Murukeshan, and R. S. Sirohi, "Separation of the influence of in-plane displacement in multiaperture speckle shear interferometry," Opt. Eng. 33, 1973 (1994).

5. N. K. Mohan and R. S. Sirohi, "Fringe formation in symmetric threeaperture speckle shear interferometry: an analysis," Opt. Lasers Eng. 26, 437 (1997)

6. N. K. Mohan and R. S. Sirohi, "Fringe formation in multiaperture speckle shear interferometry," Appl. Opt. 35, 1617 (1996).

7. H. Reismann, Elastic Plates: Theory and Application, p. 121, WileyInterscience, New York (1988).

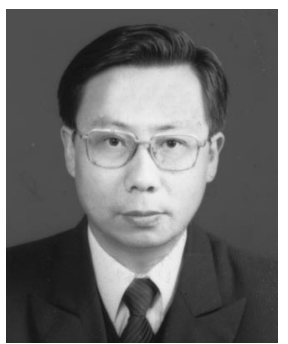

K. F. Wang received his BSc in physics from Soochow University in 1986 and his MEng in solid mechanics from Jiangsu University of Science and Technology in 1989. $\mathrm{He}$ is currently an associate professor in the Department of Mechanical Engineering, Yangzhou University. His research interests include modern photomechanics and its applications, with an emphasis on speckle techniques and their applications in engineering. He has published more than 30 publications in the field of modern photomechanics. He was given the titles of the Excellent Teacher of Jiangsu Province Higher Education and the Advanced Individual of Yangzhou University Science \& Technology in 1996. He is a member of Chinese Society of Mechanics.

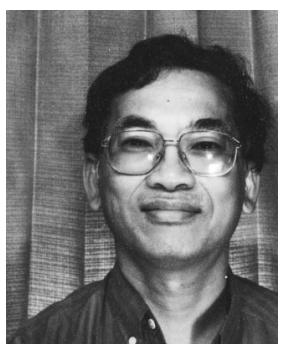

A. K. Tieu obtained his $\mathrm{PhD}$ from University of Western Australia in 1975. He was an investigation engineer with BHP Steel from 1974 to 1982 . He joined the University of Wollongong in 1982 and is now a professor of mechanical engineering.

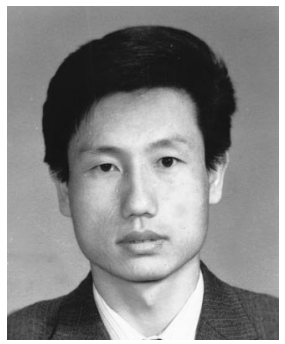

E. B. Li received his MEng and $\mathrm{PhD}$ degrees in optoelectronics from Tianjin University, China, in 1987 and 1990, respectively. From 1990 to 1994 , he worked on laser diagnostics and its applications in experimental fluid mechanics at Tianjin University. In 1994 he joined the faculty of engineering of the University of Wollongong as a research fellow. His current research interests include laser Doppler velocimetry (LDV), particle image velocimetry (PIV), and speckle metrology. 